\title{
Computer Insight into the Molecular Level of Heart Failure. What is the Role of NCX?
}

\author{
M. Fischer, M. Mlček, S. Konvičková, O. Kittnar
}

Though congestive heart failure is a leading cause of death in our population, the pathophysiological mechanisms at molecular level remain to be elucidated. This paper discusses the contribution of NCX to the pathological pattern of intracellular calcium regulation and contraction on the basis of computer simulations of a virtual cell. The model comprises calcium handling mechanisms, troponin control and acto-myosin interactions. The contribution of NCX was studied by changing its activity and turning it off for some simulations.

It was found that NCX helps to support diastolic function by reducing the Ca2+level during the diastole. At the same time there is a reduction in peak Cai and hence contraction. However, increased NCX activity does not seem to improve calcium handling and contraction crucially, as has been suggested by some authors.

Keywords: heart failure, numerical models, calcium, NCX.

\author{
Abbreviations \\ NCX $\quad \mathrm{Na}^{+/} \mathrm{Ca}^{2+}$ exchanger \\ SR sarcoplasmic reticulum \\ SERCA SR $\mathrm{Ca}^{2+}$ influx channel \\ ECC excitation-contraction coupling \\ NF non-failing heart \\ F failing heart \\ AM actomyosin complex
}

\section{Introduction}

The number of known molecular of cardiac excitation-contraction coupling (ECC) mechanisms is enormous and is still growing. It has become impossible to intuitively assess the relative contribution of an individual arrangement in physiological and pathophysiological events. However, such understanding is essential for identifying strategies for treating various types of heart failure. Mathematical modeling and simulation offers a way of handling such an extensive set of data, while still allowing for a (semi)quantitative study. Of course, there are limitations to such an approach, mainly: the accuracy of the model, its complexity (always drastically lower than in reality), low availability of consistent experimental input data, etc. However, calculations and simulations have been already proven to be useful in biological research. As an example, let us mention the correct of Na-K exchanger stoichiometry $3: 1$, based on simulations prediction as early as 1985. [1]

The aim of the present study is to assess the relative contribution of NCX and SERCA to the function of cardiac myocytes and thus to help interpret experimental data.

Generally, both proteins reduce the cytoplasmic concentration of $\mathrm{Ca}^{2+}$, and influence both the systolic (contraction) and the diastolic (relaxation) function. While SERCA transports $\mathrm{Ca}^{2+}$ into the sarcoplasmic reticulum (SR) and so increases intracellular calcium stores, i.e. the systolic function, NCX removes $\mathrm{Ca}^{2+}$ across the cellular membrane out of the cell (Fig. 1), so that the total cellular calcium is reduced and thus the systolic function is decreased. The role of NCX is far more complex, since it is regulated by voltage and by $\mathrm{Ca}^{2+}$ and $\mathrm{Na}^{+}$concentration. Consequently, it can operate in so-called "reverse mode" during the early phase of action potential.

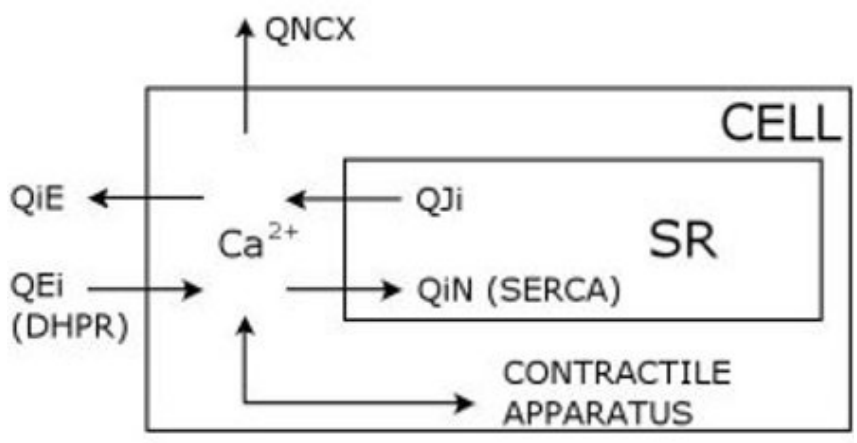

Fig. 1: Some $\mathrm{Ca}^{2+}$ transport mechanisms in heart cell

\section{Heart failure}

Congestive heart failure is an important medical issue with very high mortality and no targeted treatment available. Though it is among the leading causes of death in our population, the underlying pathophysiological mechanisms at molecular level remain to be elucidated. Altered calcium $\left(\mathrm{Ca}^{2+}\right)$ handling is seen as a key factor in the pathophysiology of heart failure. Typically, SERCA activity is consistently reported to be reduced in cases of advanced cardiac dysfunction $[2,3]$. The $\mathrm{Na}^{+} / \mathrm{Ca}^{2+}$ exchanger (NCX) has also been reported to be altered in heart failure and the currently attracting strong interest [4]. The transporter may be over-expressed [5] or normal or even reduced. The ratio between the two mechanisms seems to be an important determinant of overall function [2].

The model used for the present work is far from reproducing all known mechanisms of ECC. However, it can still provide a valuable quantitative (or semi-quantitative) insight by providing calculated data that can help to decide which are the direct effects of NCX on cellular performance (under our 
limited conditions), and which findings cannot be attributed to NCX and require identification and/or the addition of other mechanisms.

\section{Materials and Methods}

\subsection{Model}

An extended version of our earlier excitation-contraction model was used [6]. This model consists of gating, calcium, regulatory and contraction subunits (Fig. 2). A compartmental description of the underlying molecular processes is adopted to simulate a) calcium handling, b) contraction control by troponin, and c) the reaction kinetics of contractile elements. The contraction model and the calcium handling subunit were presented in [6] and [7]. Unlike the previous models, it also includes the $\mathrm{Na}^{+} / \mathrm{Ca}^{2+}$ exchanger, which was omitted or extremely simplified in earlier versions.

In principle, NCX current is now described after Winslow [8] as

$$
\begin{aligned}
I_{N a C a}(t)=k(t) & {[\mathrm{Na}]_{i}^{3} \cdot[\mathrm{Ca}]_{e} \cdot e^{(n-2) \frac{r \cdot E(t) \cdot F}{R \cdot T}} } \\
& \left.-[\mathrm{Na}]_{e}^{3} \cdot[\mathrm{Ca}]_{i}(t) \cdot e^{-(n-2) \frac{(1-r) \cdot E(t) \cdot F}{R \cdot T}}\right)
\end{aligned}
$$

and ionic flux as

$$
Q_{N a C a}(t)=\frac{A_{c a p} \cdot C_{S C}}{V_{c} \cdot z \cdot F} I_{N a C a}(t),
$$

where

$\mathrm{k}(t) \quad$ is the saturation parameter (see [8]),

$N a_{i} \quad\left(12 \mathrm{~mol} / \mathrm{m}^{3}\right)$ is intracellular sodium concentration,

$C a_{e} \quad(1,8 \mathrm{~mol} / \mathrm{m} 3)$ is extracellular calcium concentration,

$n$ (3) is stoichiometry of Na-Ca exchange,

$r(0.15)$ is position of the peak of the energy barrier separating two states - activation and deactivation of the exchanger,

$E(t) \quad$ is action potential,
$F \quad(96485.3415 \mathrm{~s} \cdot \mathrm{A} / \mathrm{mol})$ is Faraday constant,

$R \quad(8.3144 \mathrm{~J} / \mathrm{K} \cdot \mathrm{mol})$ is ideal gas constant,

$T \quad(290.15 \mathrm{~K})$ is absolute temperature,

$\mathrm{Na} a_{e} \quad\left(140 \mathrm{~mol} / \mathrm{m}^{3}\right)$ is extracellular sodium concentration, $C a_{i}(t)$ is intracellular calcium concentration,

$A_{\text {cap }} \quad\left(1.534 \mathrm{e}^{-8} \mathrm{~m}^{2}\right)$ is capacitive membrane area,

$C_{S C} \quad\left(1 \mathrm{~F} / \mathrm{m}^{2}\right)$ is specific membrane capacity,

Vc $\quad\left(25 \mathrm{e}^{-12} \mathrm{~m}^{3}\right)$ is cellular volume, and

$z(2)$ is valence of calcium ion.

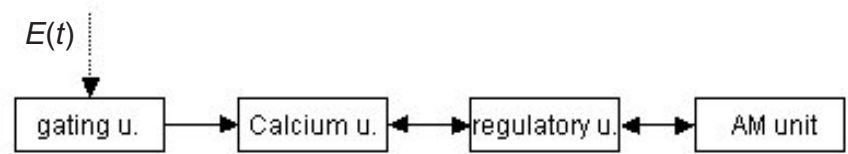

Fig. 2: Model scheme - subunits interaction

\subsection{Simulation and parameters}

The model was implemented in Matlab/Simulink. The ode15s (stiff/NDF) integration method was used. As an input, the experimentally obtained trace of cardiac action potential was used (Fig. 3) [9]. Simulation parameters: stimulation frequencies 1.2 and $3 \mathrm{~Hz}$, simulation duration $300 \mathrm{~s}$ (to avoid transient state effects).

The model was also verified for various action potential durations (APD) in accordance with reality where APD varies physiologically both with heart rate and in various regions of the myocardium. However, due to the simplicity, of the model, it currently does not essentially reflect any mechanisms influencing the shaping and action potential duration, such as modification of the activity of ionic channels.

Heart failure model: Chronic congestive failure was simulated according to experimental findings [10] by reducing the activity of SERCA from $100 \%$ to 75 and $50 \%$ of normal values. SERCA ionic flux is defined by the equation

$$
Q_{i N}(t)=\left(C a_{i}(t)-C a_{i 0}\right) \cdot\left(k_{i N}+k_{i N a c t} \cdot f_{\infty}(t)\right),
$$

where

$Q_{i N}(t)$ is ionic flux from the intracellular space to the network sarcoplasmic reticulum (NSR),

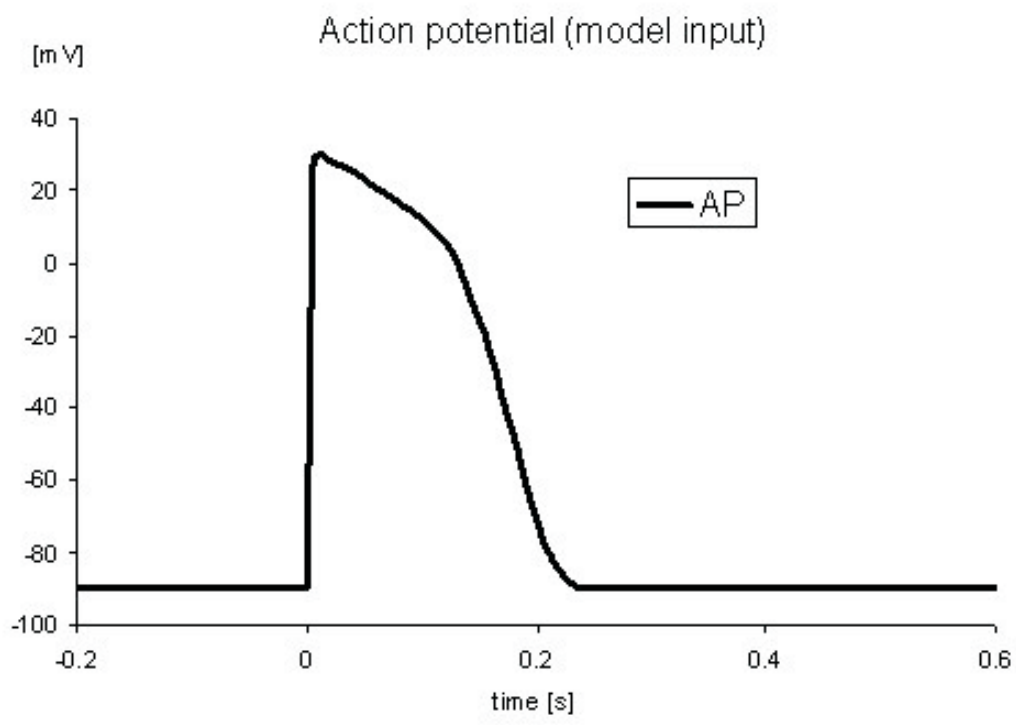

Fig. 3: Action potential - model input 
$C a_{i 0} \quad\left(1 \mathrm{e}^{-4} \mathrm{~mol} / \mathrm{m}^{3}\right) \quad$ is initial intracellular calcium concentration,

$k_{i N} \quad\left(4 \mathrm{~s}^{-1}\right)$ is the rate constant of the passive part of SERCA channel,

$k_{\text {iNact }}\left(1 \mathrm{~s}^{-1}\right)$ is the rate constant of the active part of the SERCA channel, and

$f_{\infty}(\mathrm{t}) \quad$ is steady-state deactivation coefficient.

According to some findings that report NCX activity in failing hearts [11], the NCX activity was varied between 50 , 75,100 and $130 \%$ of normal activity. The NCX transporter was also virtually completely blocked (activity set to $0 \%$ ), so that its (missing) role in ECC could be identified more easily.

\section{Results}

\subsection{NCX in a non-failing heart (NF)}

Though NCX is a minor calcium-removal mechanism (10 25\% of total Ca to be removed), its contribution to calcium handling is substantial and becomes obvious over a period of time (here, after $300 \mathrm{~s}$ at $1 \mathrm{~Hz}$ ). The absence of NCX activity (Fig. 4) results in more $\mathrm{Ca}^{2+}$ remaining in the cell. This calcium is transported into internal stores (the sarcoplasmic reticulum) by the SERCA transporter. Even the rather small amount of $\mathrm{Ca}^{2+}$ that is retained during each beat gradually loads the internal calcium stores until a new input-output

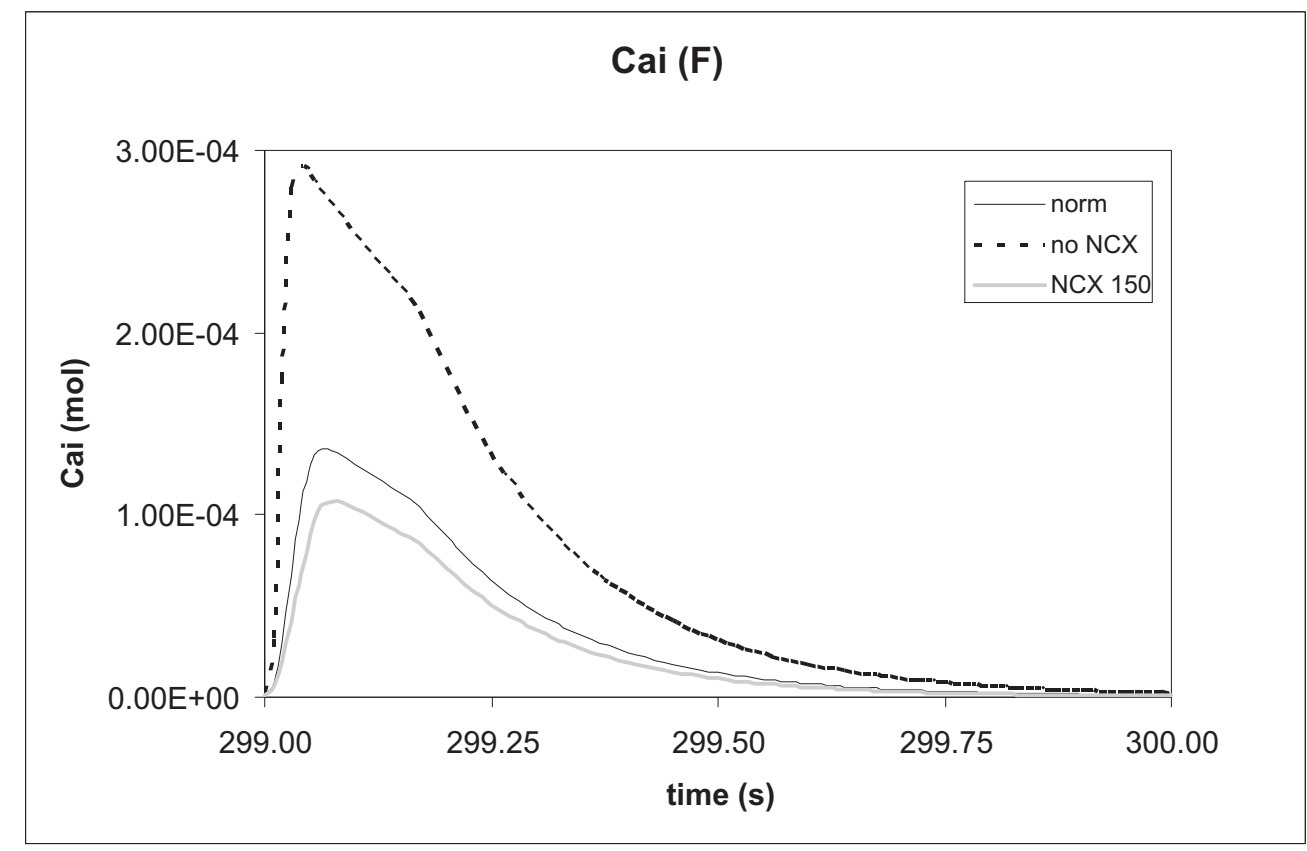

Fig. 4: Intracellular $\mathrm{Ca}^{2+}$ concentration in NF and F heart (NCX activity $0 \%$ and $150 \%$ ), at $1 \mathrm{~Hz}$

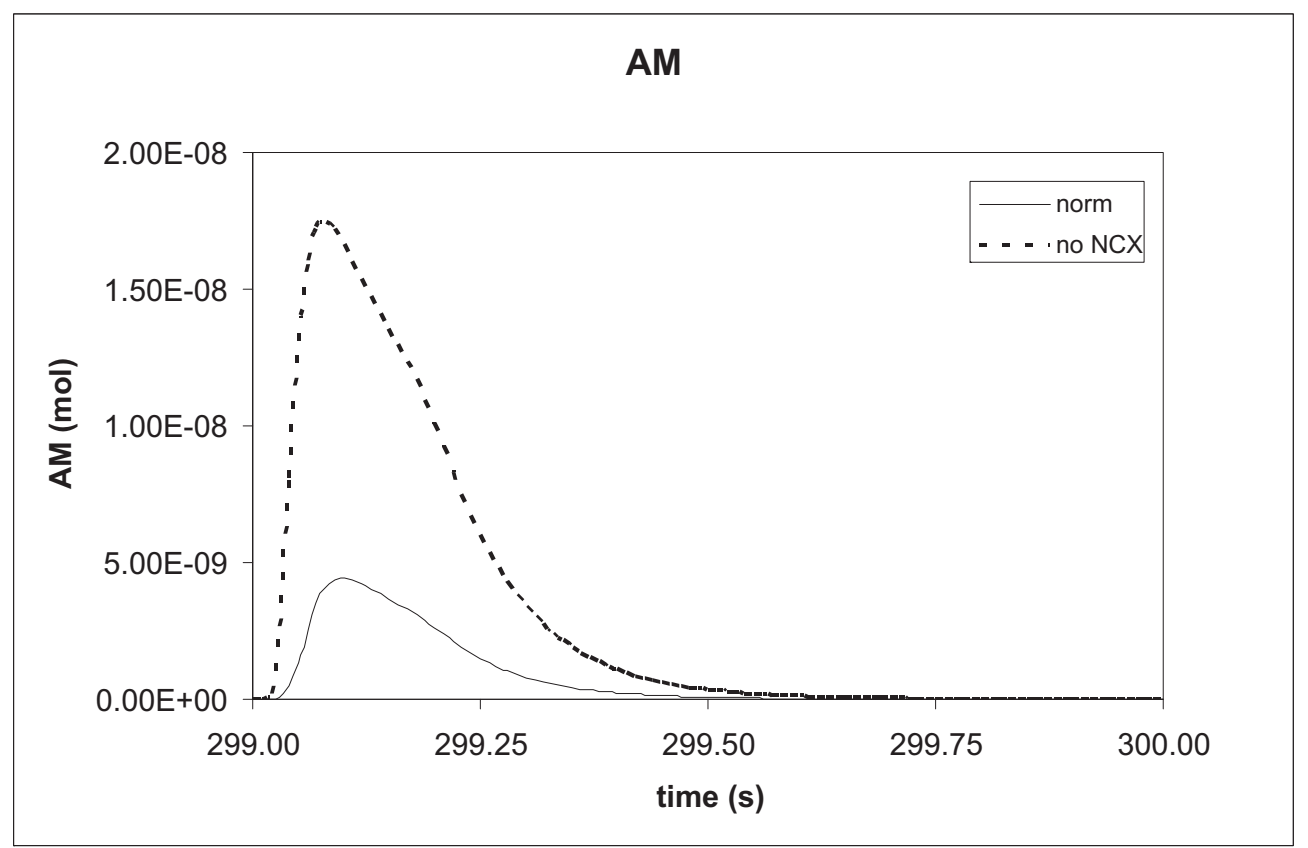

Fig. 5: Contractile force in NF heart and in heart with turned NCX off, at $1 \mathrm{~Hz}$ 


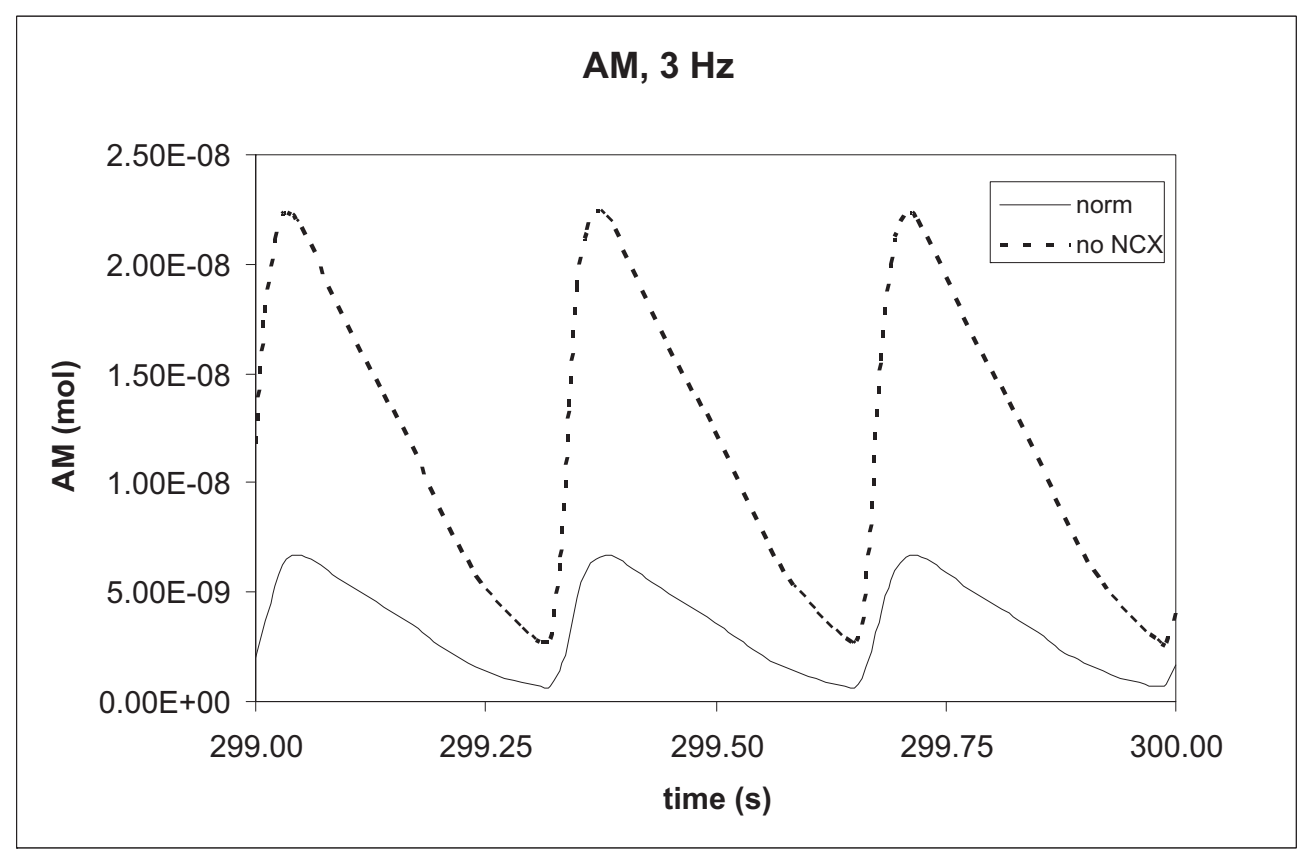

Fig. 6: Contractile force in NF heart and in heart with turned NCX off, at $3 \mathrm{~Hz}$

equilibrium is reached. Thus during activation more $\mathrm{Ca}^{2+}$ will be released and so more force will be generated. At the same time, the relaxation of contraction is prolonged. Increased NCX activity exhibits opposite effects.

Thus it can be concluded that increased NCX activity (the forward mode) alone decreases the force (negative inotropic effect) and improves relaxation (positive lusitropic effect), and vice versa.

The kinetics of an acto-myosin strong interaction $(A M(t))$ represents the mechanical performance of virtual cardiac myocyte. This is obtained from the model simulation (contraction subunit). The original description was given in [6].

\section{The effect of heart rate}

The effect of NCX during each cardiac cycle is time-limited, and is therefore directly influenced by heart frequency. Shortening the cardiac period means that less time is available for $\mathrm{Ca}^{2+}$ removal by NCX (Fig. 8). If NCX activity is decreased, at higher rates $(2-3 \mathrm{~Hz}) \mathrm{Ca}^{2+}$ does not return to

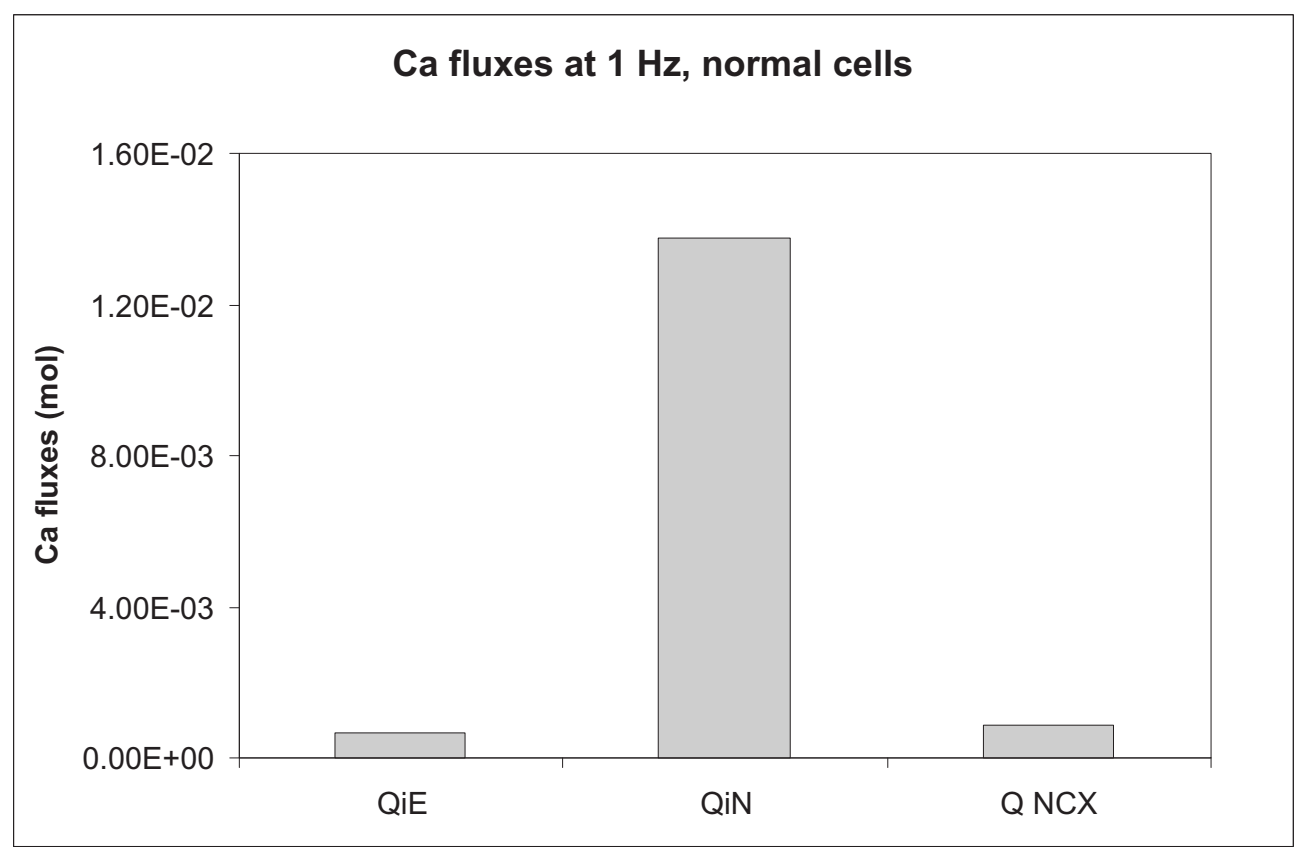

Fig. 7: Total $\mathrm{Ca}^{2+}$ fluxes (per $1 \mathrm{sec}$ ) at $1 \mathrm{~Hz}, \mathrm{NF}$ 


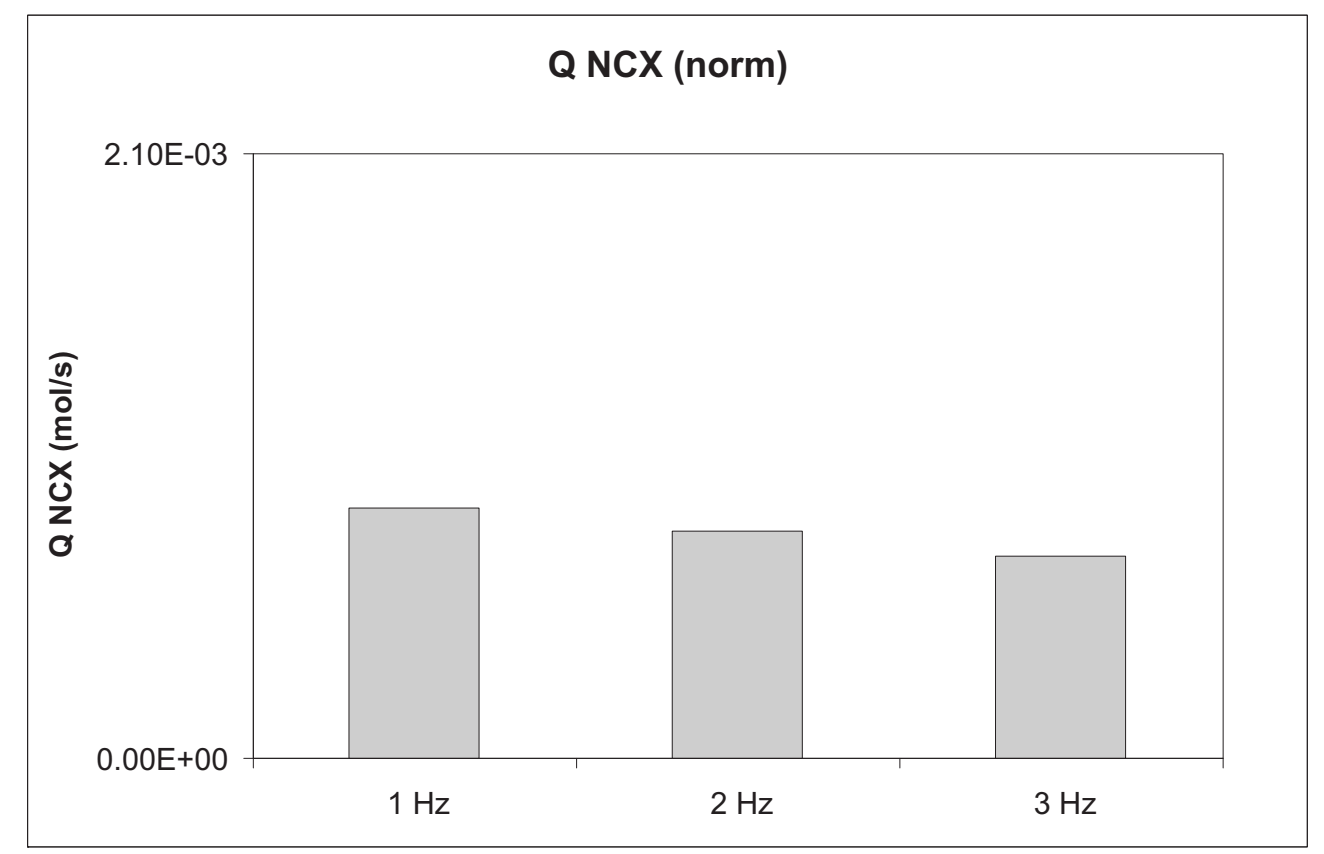

Fig. 8: Total $\mathrm{Ca}^{2+}$ fluxes (per $1 \mathrm{sec}$ ) at 1,2 and $3 \mathrm{~Hz}, \mathrm{NF}$

the baseline, and therefore the myocardium may not relax properly between beats (Fig. 6).

\subsection{NCX in SERCA dysfunction}

Impaired calcium handling in chronic heart failure $(\mathrm{F})$ was simulated by decreasing the SERCA activity to $50 \%$ of the norm. The activity of NCX was varied between 0,100 and $150 \%$. Decreased SERCA activity alone resulted in lower calcium peak and force transient (by cca 40\%) and increased calcium during the relaxation phase (compared to the non- -failing model). This is consistent with many experimental findings $[11,12]$. By increasing the activity of NCX to $150 \%$ of $\mathrm{N}$, the relaxation (force and $\mathrm{Ca}^{2+}$ ) was restored. However, this is at the cost of a further decline of the maximal force (to $50 \%$ ) (Fig. 9). Importantly, this effect was pronounced at high frequencies $(3 \mathrm{~Hz}-$ Fig. 10).

\section{Discussion}

The role of NCX in ECC is quite complex. At first, by removing calcium from the cell, increased NCX reduces

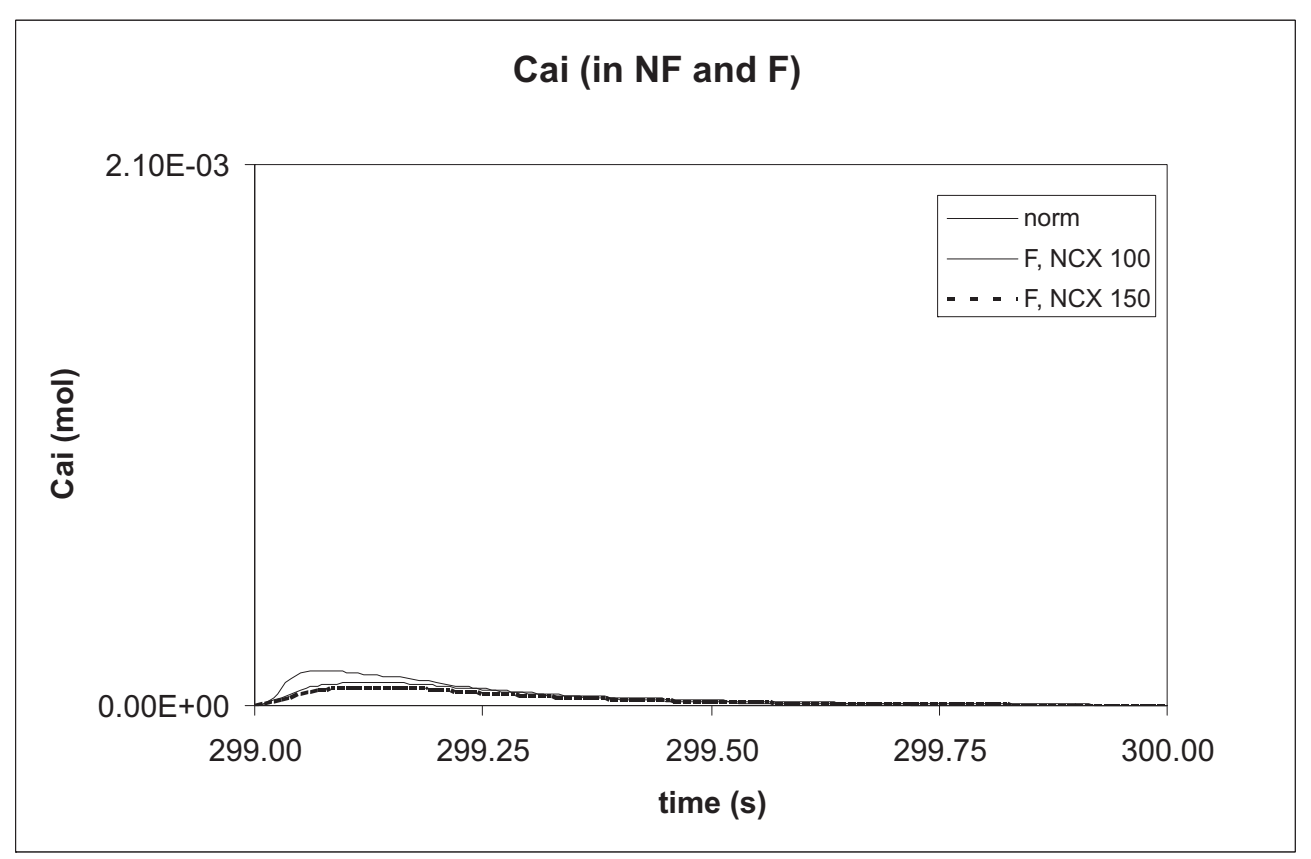

Fig. 9: Intracellular $\mathrm{Ca}^{2+}$ concentration in NF and $\mathrm{F}$ heart (SERCA activity $50 \%$, NCX activity $100 \%$ and $150 \%$ ), at $1 \mathrm{~Hz}$ 


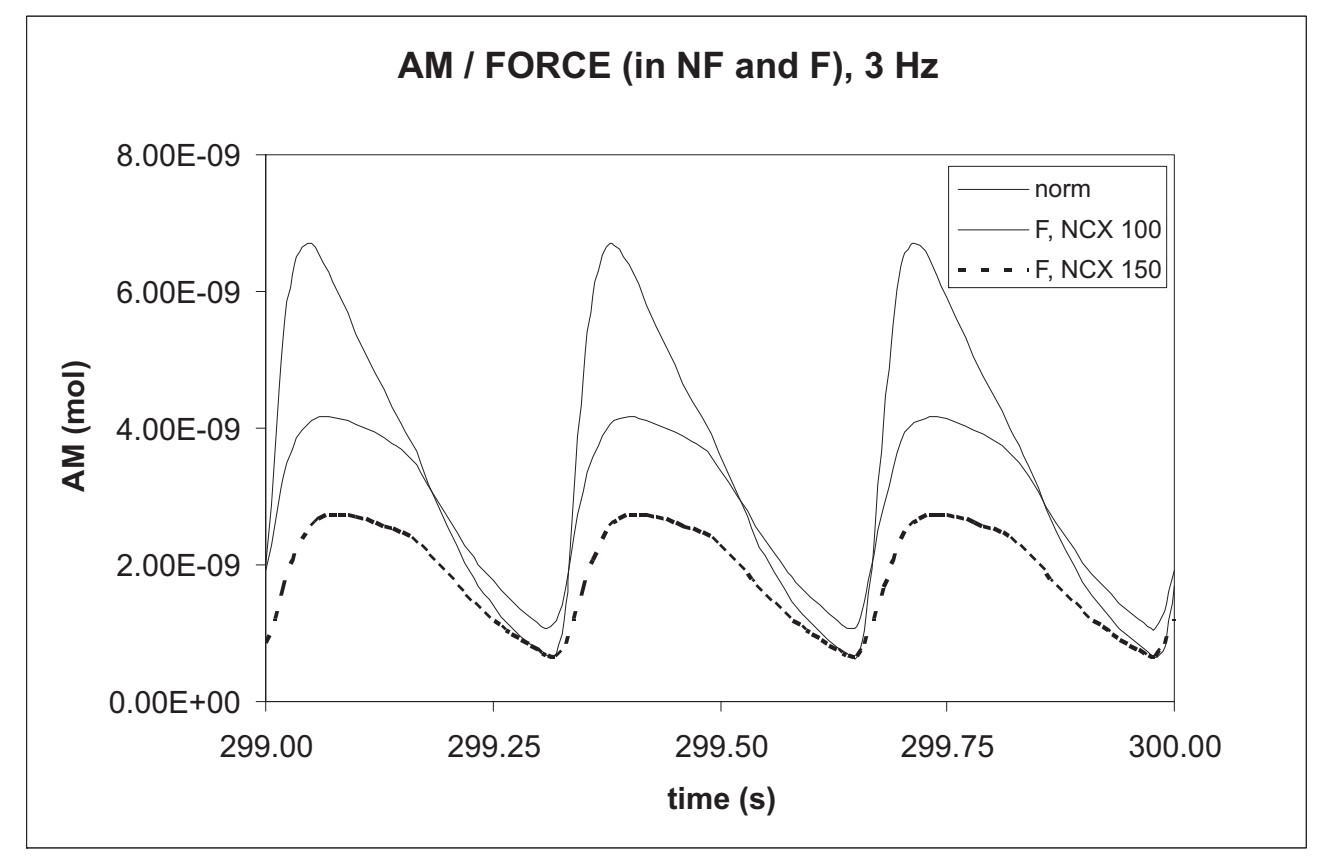

Fig. 10: Contractile force in NF and F heart (SERCA activity $50 \%$, NCX activity $100 \%$ and $150 \%$ ), at $3 \mathrm{~Hz}$

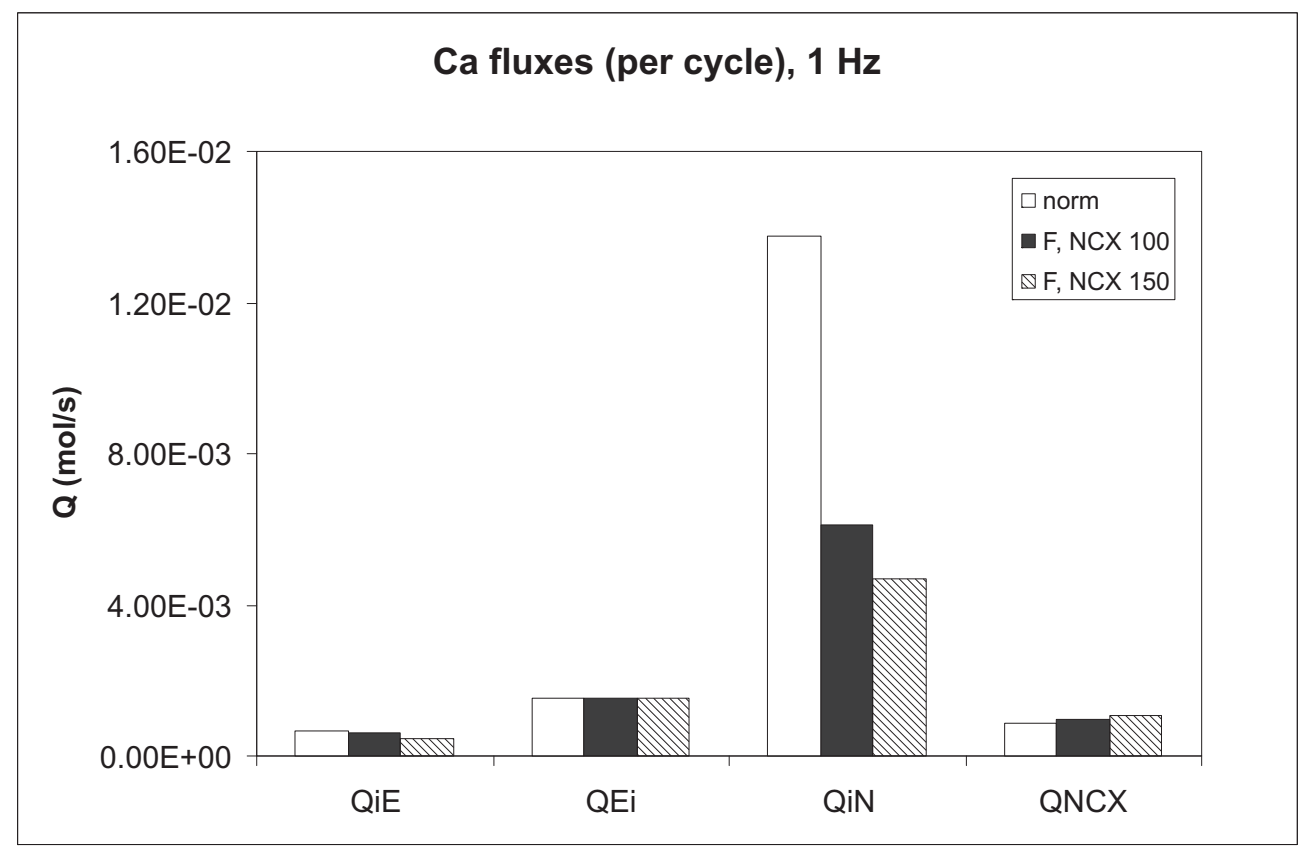

Fig. 11: Total $\mathrm{Ca}^{2+}$ fluxes (per $1 \mathrm{sec}$ ) in NF and F heart (SERCA activity $50 \%$, NCX activity $100 \%$ and $150 \%$ ), at $1 \mathrm{~Hz}$

contractility and thus could have a negative effect on cardiac performance. This is in line with some experimental findings [13]. Though the transporting capacity of NCX is much lower than that of other mechanisms (SERCA), NXC can influence the gradual loading/unloading of cellular calcium stores. Cellular calcium determines the maximal contraction force. Secondly, lowering cellular calcium (both in stores and free $\mathrm{Ca}^{2+}$ ) has a direct positive effect on cardiac relaxation and so can improve heart performance - the heart is a pulsation pump so the relaxation is as necessary as the contraction and both need to alternate periodically. Moreover, the nutrition (blood supply) of the heart is better during the relaxation phase (it almost stops during peak contraction). Thus it cannot be simply concluded which of the two effects should take precedence. Nor can we calculate the amplitude of force (AM) oscillation as a measure of performance. Thirdly, NCX is capable of "reverse mode" operation, i.e. it can load calcium into the cell during the early phase of the cardiac cycle. The effect of NCX is also heart-rate dependent. At low frequencies $(<\sim 1 \mathrm{~Hz})$, the relatively long period offers enough reserve capacity for extrusion of $\mathrm{Ca}^{2+}$, which controls relaxation. The extrusion (and therefore the relaxation) takes longer, but ultimately fully completes (Figs. 4, 5). At higher rates full relaxation is not achieved. 
In heart failure, reduced SERCA is consistently reported. In line with this, lower total calcium, calcium peak and maximal force are found. However, reports on the role of NCX are almost contradictory. O'Rourke [11] reports increased NCX that compensates defective SERCA in maintaining diastolic (relaxed) calcium low at the cost of further compromised systolic (contraction) performance. Based on our results, this compensatory effect is relevant mainly when the heart rate is higher.

However, Hobai [12] reports improved cardiac function in a failing heart after inhibition of increased NCX by externally administered inhibitory peptide, and suggests NCX inhibition as a promising treatment for heart failure. This finding may also be relevant, however only when the heart rate is low (circa $1 \mathrm{~Hz}$ and less). Blocking NCX: a) increases cellular calcium loading (and contractility), b) increases diastolic $\mathrm{Ca}^{2+}$. At low heart rates, there is enough time to extrude this increased $\mathrm{Ca}^{2+}$ (Fig. 9) and so maintain relaxation. At low rates overall stimulation predominates. At rates $\geq 2 \mathrm{~Hz}$ diastolic $\mathrm{Ca}^{2+} \mathrm{ac}-$ cumulates, since it is not sufficiently extruded and results in incomplete relaxation (Fig. 10). The experiments by Hobai were performed at $0.5 \mathrm{~Hz}$ which may explain why they reported a generally beneficial role of NCX inhibition. However, in clinical cases there could be double-edged effects, since the heart frequency can easily reach $2 \mathrm{~Hz}$.

Some other works have suggested increased cardiac reserve performance after over-expression of NCX in a failing rabbit heart [13]. This effect cannot be explained by the current model, and thus requires further mechanisms to be identified and included.

No matter how complex calcium handling is, it must be true that the input and output of $\mathrm{Ca}^{2+}$ into and out of the cytoplasm must generally match. Any change in one of the transporting mechanisms (SERCA, NCX, others) must be reflected by changes in other parameters (cellular $\mathrm{Ca}^{2+}$ stores, free $\mathrm{Ca}_{\mathrm{i}}^{2+}$, etc.) The major role of NCX is in removing $\mathrm{Ca}^{2+}$, which (necessarily) enters the cell through an L-type channel (DHPR) from outside during each cycle. Thus increased NCX activity will always tend to unload the cell and compromise heart contractility. Increased NCX $200 \%$ combined with reduced SERCA $50 \%$, as sometimes reported in heart failure, would result in extreme depletion of internal calcium and thus the force that could be generated is hardly sufficient. This calculation indirectly suggests that another mechanism must be involved in $\mathrm{Ca}^{2+}$ handling changes in heart failure. One option is the "reverse mode" capability of NCX in the early phase of a cycle. This regime depends on many parameters $\left(\mathrm{Na}^{+}\right.$and $\mathrm{Ca}^{2+}$ concentrations, membrane voltage, $\mathrm{NCX}$ regulations) and plays a minor role under physiological conditions. However, to make a quantitative assessment of this effect and its contribution to cell $\mathrm{Ca}^{2+}$ handling as a whole, specifically in a simulated failing heart, the current model needs to be refined to better reflect the parameters controlling the NCX reverse mode.

\section{Limitations}

In biological systems numerous molecular mechanisms contribute to ECC. Any of them can be altered and many may even not have been identified yet. Conversely, a computational model can only reflect a very limited set of the mechanisms that are included in the real phenomenon in real life.
The results drawn here should always be interpreted with caution. However, good conformance with experimental data indicates that major mechanisms have been identified (e.g. the effect of NCX at various heart rates). However, the discrepancy between the simulated and experimental data suggests that further mechanisms need to be searched for (e.g. improved contractility after NCX stimulation in healthy hearts). In this way, simulations can stimulate and direct biomedical research, which has traditionally relied on experimental approaches.

\section{Acknowledgment}

This study was supported by the Ministry of Education, Youth and Sports of the Czech Republic project: Transdisciplinary research in Biomedical Engineering II., No. MSM 6840770012 and the Grant Agency of the Czech Republic, project No. 106/04/1181, and Czech Academy of Sciences 1ET201210527.

\section{References}

[1] Di Francesco, D. - Noble, D.: A Model of Cardiac Electrical Activity Incorporating Ionic Pumps and Concentration Changes - Simulations of Ionic Currents and Concentration Changes. Philos.Trans. R. Soc. Lond B Biol. Sci., 1985, vol. 307, p. 353-398.

[2] Hasenfuss, G. et al.: Relationship between $\mathrm{Na}^{+}-\mathrm{Ca}^{2+}$-exchanger Protein Levels and Diastolic Function of Failing Human Myocardium. Circulation, 1999, vol. 99, p. 641-648.

[3] Huke, S. et al.: Altered Force-Frequency Response in Non-Failing Hearts with Decreased SERCA Pump-Level. Cardiovasc. Res., 2003, vol. 59, p. 668-677.

[4] Hasenfuss, G. - Schillinger, W.: Is Modulation of Sodium-Calcium Exchange a Therapeutic Option in Heart Failure? Circ.Res., 2004, vol. 95, p. 225-227.

[5] Pieske, B. et al.: Functional Effects of Endothelin and Regulation of Endothelin Receptors in Isolated Human Nonfailing and Failing Myocardium. Circulation, 1999, vol. 99, p. 1802-1809.

[6] Mlček, M. et al.: Mathematical Model of the Electromechanical Heart Contractile System-Regulatory Subsystem Physiological Considerations. Physiol Res., 2001, vol. 50, p. 425-432.

[7] Novak, V. - Neumann, J.: Mathematical Model of the Electromechanical Heart Contractile System - Simulation Results. International Journal of Bioelectromagnetism, 2000, vol.2, no. 2, electronic version.

[8] Winslow, R. L. et al.: Mechanisms of Altered Excitation-Contraction Coupling in Canine Tachycardia-Induced Heart Failure, II: Model Studies. Circ.Res., 1999, vol. 84, p. 571-586.

[9] Trautwein, W. - Kassebaum, D. G... Electrophysiological Study of Human Heart Muscle. Circ Res, 1962, vol. 10, p. 306-312.

[10] Beuckelmann, D. J. - Nabauer, M. - Erdmann, E.: Intracellular Calcium Handling in Isolated Ventricular Myocytes from Patients with Terminal Heart Failure. Circulation, 1992, vol. 85, p. 1046-1055. 
[11] O'Rourke, B. et al.: Mechanisms of Altered Excitation-Contraction Coupling in Canine Tachycardia-Induced Heart Failure, I: Experimental Studies. Circ.Res., 1999, vol. 84, p. 562-570.

[12] Hobai, I. A. - Maack, C. - O'Rourke, B.: Partial Inhibition of Sodium/Calcium Exchange Restores Cellular Calcium Handling in Canine Heart Failure. Circ.Res., 2004, vol. 95, p. 292-299.

[13] Munch, G. et al.: Functional Alterations after Cardiac Sodium-Calcium Exchanger Overexpression in Heart Failure. Am. J. Physiol Heart Circ.Physiol, 2006, vol. 291, p. H488-H495.

Martin Fischer, MSc.

phone: +420224352542

e-mail: mail@martinfischer.cz

Department of Mechanics, Biomechanics and Mechatronics

Czech Technical University

Faculty of Mechanical Engineering

Technická 4

16607 Prague 6, Czech Republic
Mikuláš Mlček, MD, Ph.D.

phone: +420224968407

e-mail: mikulas.mlcek@lf1.cuni.cz

Institute of Physiology

Charles University

First Faculty of Medicine

Albertov 5

12800 Prague 2, Czech Republic

Svatava Konvičková, MSc, Ph.D.

phone: +420224352511

e-mail: svatava.konvickova@fs.cvut.cz

Department of Mechanics, Biomechanics and Mechatronics

Czech Technical University

Faculty of Mechanical Engineering

Technická 4

16607 Prague 6, Czech Republic

Otomar Kittnar, MD, Ph.D.

phone: +420224912903

e-mail: otomar.kittnar@1f1.cuni.cz

Institute of Physiology

Charles University

First Faculty of Medicine

Albertov 5

12800 Prague 2, Czech Republic 